\title{
Création et évolution d'un tutorat en première année du premier cycle des études médicales
}

\section{Un projet étudiant devenu «institution», chronique d'une «success story"}

\author{
Implementing and developing free student tutoring in the first year \\ of the pre-graduate curriculum
}

\author{
A formalized student project that has turned into a success story \\ Guillaume LeBreton, Jean-Philippe GIOT et Régis DELAUnAY \\ Université de Caen, Basse-Normandie, 50530 Dragey, France
}

Manuscrit reçu le 19 octobre 2009 ; accepté pour publication le 26 juillet 2010

\begin{abstract}
Mots clés :
Premier cycle des études médicales ; tutorat ;

projet étudiant

Résumé - Contexte et problématique : La sélectivité du concours de fin de première année des études médicales en France, associée aux bouleversements que constitue l'entrée dans l'enseignement supérieur, confère au cursus de la première année du premier cycle des études médicales (PCEM1) un caractère particulièrement ardu. Des instituts privés proposent des formations coûteuses visant à aider les étudiants à mieux appréhender ce cap difficile. Exégèse : Nous rapportons une expérience de dix années d'un tutorat gratuit créé par des étudiants. Ce tutorat est devenu une «institution » pérenne et offre un soutien régulier et efficace aux étudiants de PCEM1. De plus, il offre aux «tuteurs» une formation originale et une valorisation de leur engagement, au travers d'une option qui constitue la clé de voûte de ce tutorat et en explique le succès.
\end{abstract}

Keywords:

Pre-graduate curriculum; peer teaching; student project

Abstract - Context and issues: In addition to the majors changes associated with taking on graduate studies, the selection requirements of the exam at the end of the first year of French pre-graduate medical training (PCEM1) are especially challenging with respect to this curriculum, even though private institutions offer high-priced training to help students get over this hurdle. Exegesis: Some 10 years of free peer-teaching created by students have provided valuable experience in this area. The mentoring has become a permanent "institution" offering recurring and effective support to PCEM1 students. Through an alternative that is a cornerstone for peer-teaching and explains its success, "tutors" acquire unique training and raise their level of commitment. 
Répondant à l'invitation formulée dans l'éditorial de février 2008, qui encourageait les étudiants à partager leurs témoignages d'expériences d'enseignement et d'apprentissage ${ }^{[1]}$, nous souhaitons rapporter notre expérience d'un projet pédagogique initié depuis une dizaine d'années par des étudiants à la faculté de médecine de Caen (Université de CaenBasse Normandie, France) et qui s'est ultérieurement consolidé au point de devenir une «institution ».

\section{L'origine du tutorat à la faculté de médecine de Caen}

Actuellement en cours d'internat, nous avons mis en place à Caen, lorsque nous étions externes, un système de tutorat en première année, afin d'aider les étudiants à mieux appréhender ce cap difficile qu'est le concours de fin de première année des études médicales (PCEM1). On rappelle que le classement en rang utile à ce concours conditionne la possibilité de poursuivre ses études médicales, qui sont encadrées en France par un numerus clausus, déterminé chaque année par un arrêté du ministère de la santé. En 1998, lorsque nous étions nous-mêmes en cours de préparation de ce concours, aucun système de ce genre n'existait. Un institut de formation privé proposait une formation fort coûteuse. L'année suivante, trois instituts de ce genre ouvraient leurs portes et faisaient salles combles. Afin de proposer une forme de soutien et de préparation au concour, les étudiants de deuxième année des études médicales décidèrent de mettre en place un concours d'entraînement ou « concours blanc ». Celui-ci rencontra un vif succès auprès des étudiants et reçut un franc soutien de la part des enseignants.

La réalisation d'une épreuve «blanche» ne nous semblait pas suffisante. De plus, l'idée qu'une inscription à des instituts privés de préparation au concours devienne un passage obligé pour réussir le concours de PCEM1, constituant de fait une forme de sélection par l'argent, source d'inégalité des chances, nous incita à réfléchir à d'éventuels prolongements à l'initiative. Ainsi, en 2001, forts de cette expérience, nous décidions de compléter l'aide proposée aux étudiants pour préparer le concours sous la forme d'un tutorat. À cette fin, une association, dénommée «Association du tutorat en médecine de Caen », fut crée. Cette association avait - et a toujours - pour but d'organiser gratuitement un soutien plus complet, sous forme de séances d'entraînement hebdomadaires, dites «colles », et de développer les liens entre les étudiants de première année et leurs aînés.

\section{L'organisation du tutorat}

Nous souhaitions d'une part que le soutien apporté aux étudiants s'exerce à l'occasion d'activités se rapprochant le plus possible des épreuves du concours; nous souhaitions également que les exigences en termes d'accaparement du temps soient raisonnables et que les activités de soutien ne soient pas trop «chronophages» pour les étudiants de PCEM1. Aussi, avons nous opté pour des «colles » hebdomadaires, avec des exercices calqués sur les épreuves du concours, rédigés et corrigés par les tuteurs et relus par les enseignants. En outre, dans la perspective de la préparation à un concours classant, il est nécessaire de fournir aux étudiants des moyens leur permettant de comparer leurs performances à celles de leurs pairs; c'est en cela que nous pouvions apporter un plus par rapport aux instituts privés. À l'issue de chaque colle, un classement virtuel et anonyme était effectué et diffusé sur le site internet du tutorat, que nous avions créé et développé et qui constituait un outil indispensable.

Ce tutorat remporta un franc succès et fit d'ailleurs l'objet de plusieurs reportages télévisés, sur la chaîne régionale France 3 Normandie. Une enquête réalisée en mars 2002 auprès des étudiants de PCEM1 montrait leur très grande satisfaction mais des critiques liées à la qualité variable des différentes colles étaient formulées. De plus, la motivation des tuteurs, tous étudiants de deuxième année des études médicales (PCEM2), volontaires et bénévoles, semblait s'essouffler au fil de l'année, en raison notamment de la charge de travail importante que cela impliquait. Nous avons alors eu le sentiment que, malgré des débuts prometteurs, notre 
projet était vulnérable et à un tournant de son existence : soit nous trouvions une solution pour lui assurer une pérennité, soit il disparaîtrait rapidement.

\section{Assurer la pérennité}

En juin 2002, nous proposions donc à la commission pédagogique de la faculté de médecine une initiative originale qui, selon nous, pouvait garantir la pérennité du système. Il s'agissait d'organiser, à partir de la rentrée 2002-2003, un certificat optionnel «tutorat ». Dans le cadre du dispositif français des études médicales, les certificats optionnels sont des unités d'enseignement et d'apprentissage auxquelles les étudiants s'inscrivent, en choisissant parmi une liste de certificats agréés; en PCEM2, chaque étudiant doit obligatoirement s'inscrire à une option de son choix. Nous postulions d'une part que la proposition d'un certificat optionnel « tutorat » donnerait un « cadre » pédagogique au tutorat et permettrait d'en améliorer la qualité ; en outre, l'intégration du tutorat comme l'une des options possibles dans le cursus régulier obligatoire de PCEM2 permettait de ne pas accroître la charge de travail des étudiants tuteurs qui faisaient ce choix, ce nous constituait à nos yeux un facteur de pérennité du dispositif. Nous proposions alors un programme d'enseignements théoriques s'articulant autour de plusieurs axes : pédagogie, technologies de l'information et de la communication, statistiques et communication. À notre grande surprise, cette initiative fut accueillie avec enthousiasme par les enseignants et l'administration et, en septembre 2002, cet enseignement optionnel fut proposé aux étudiants.

Depuis sa création, 30 étudiants de PCEM2, soit environ un tiers de l'effectif de chaque promotion, s'inscrivent chaque année à cette option qui, selon nous, est la clé de voûte de ce tutorat. Les tuteurs comme les «tutorés » en tirent un bénéfice, déterminant essentiel de la motivation tant des enseignants que des apprenants ${ }^{[3]}$. Les tuteurs ont conscience de participer à un enseignement original et tirent satisfaction de la reconnaissance et de la valorisation de leur travail; les «tutorés », de leur côté, bénéficient d'un soutien efficace, régulier et de qualité.

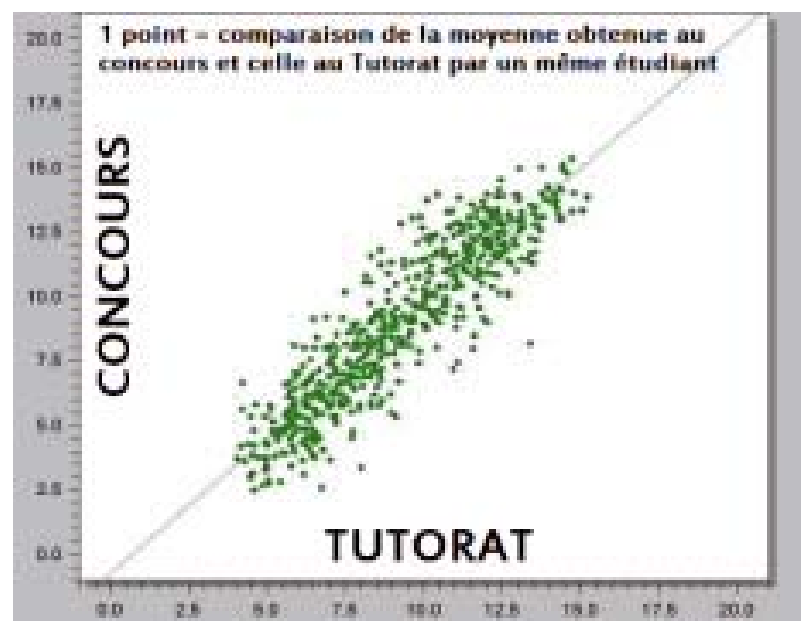

Fig. 1. Corrélation entre les scores obtenus au concours de fin de première année des études médicales et les scores obtenus aux exercices organisés dans le cadre du tutorat (Dr Creveuil - statisticien - CHU de Caen).

\section{Le tutorat en 2009}

À l'heure actuelle, le tutorat est devenu un dispositif incontournable de la PCEM1 à la faculté de médecine de Caen, avec un taux d'inscription proche de $100 \%$ en début d'année et une participation qui se stabilise à $80 \%$ des inscrits au fil des mois. L'évaluation des performances des étudiants dans le cadre des colles est fiable. Une étude statistique réalisée en 2006 sur 692 étudiants a montré qu'une forte corrélation existait entre les scores obtenus aux exercices proposés lors des colles et ceux obtenues aux épreuves du concours (figure 1) et que les étudiants inscrits au tutorat obtenaient en moyenne un score au concours supérieur de 2 points sur 20, par rapport à celui des non-inscrits $(p<0,001)$. Selon nous, de tels résultats contribuent fortement à la crédibilité du dispositif et l'efficacité de ce soutien gratuit offert par le tutorat est la clé de son succès.

Devenus internes à l'issue des épreuves classantes nationales de 2004, nous avons depuis quitté la subdivision d'internat de Caen mais, en 2009, le tutorat existe toujours dans cette faculté. En dix ans, les services proposés se sont étoffés ${ }^{[2]}$ par la mise en place d'une banque des questions-réponses, par l'organisation de séances hebdomadaires de corrections et d'explications des exercices, par l'organisation 
d'un forum des métiers de la santé, par des visites des lycées et l'organisation de journées d'accueil, cette liste n'étant pas exhaustive. Le site internet du tutorat a été rénové (www . unicaen. fr/ufr/ medecine/tutorat) et constitue une interface d'échange et de communication, ainsi qu'un outil d'évaluation.

Au-delà de la préparation au concours, le tutorat est devenu un véritable accompagnement de l'étudiant de première année par ses pairs. Chaque tuteur a en charge un groupe d'étudiants qu'il suit tout au long de l'année. L'étudiant peut ainsi trouver un soutien psychologique auprès d'un aîné lorsque cela est nécessaire. De plus, en donnant à chaque étudiant la possibilité d'évaluer comparativement ses performances au fil de l'année, le tutorat lui permet d'adapter sa technique de travail, voire d'envisager une éventuelle réorientation, en «temps réel », sans attendre l'inéluctable sanction du concours ${ }^{[2]}$.

\section{Conclusion}

C'est notamment grâce à la confiance et au soutien fort que nous avons reçu de la part des enseignants et de l'administration de la faculté de médecine et de l'Université de Caen-Basse-Normandie que l'initiative du tutorat a pu voir le jour il y a dix ans et existe encore aujourd'hui, au point d'être devenue une véritable « institution » en PCEM1. L'efficacité et la qualité du soutien apporté sont illustrés par les très forts taux d'inscription et d'assiduité, constants depuis des années. Un tel dispositif aurait cependant disparu si nous n'en avions pas garanti la pérennité grâce à la création du certificat optionnel, qui contribue à sa qualité et valorise l'engagement des tuteurs. Ce sont là, selon nous, les deux facteurs clés de la pérennité de ce tutorat, qui renoue avec une longue tradition de compagnonnage dans l'enseignement de la médecine.

\section{Contributions}

Les trois auteurs ont créé le projet de tutorat à la faculté de médecine de Caen, présenté dans cet article. Ils ont solidairement rédigé le manuscrit.

\section{Remerciements :}

Nous tenons à remercier toutes les personnes ayant contribué à la réalisation de ce projet : les anciens et actuels tuteurs de deuxième année du premier cycle des études médicales (PCEM2) et leurs présidents, tout particulièrement Lisa Pellerin et Virginie Testu. Nous adressons nos remerciements aux enseignants de première année du premier cycle des études médicales (PCEM1) et notamment à Mme Laroche, MM. Allouche, Agostini, Bouvard, Charbonneau, Creveuil, Moreau, Gérard (directeur de l'UFR) et Salame, ainsi que Mmes Danièle Verrolles (directrice administrative de l'UFR) et Nicole LeQuerler (présidente d'université) pour leur soutien et leur confiance dès les premières heures.

\section{Références}

1. Jouquan J, Maillard D. «Paroles d'étudiants », une nouvelle rubrique. Pédagogie Médicale 2008;9:6.

2. Lebreton G, Giot JP, Delauney R, Gérard JL, Agostini D. PCEM1 : 10 ans de tutorat à Caen, un projet étudiant devenu institutionnel. Presse Med 2009;38:1165-6.

3. Bernard JL, Reyes P. Apprendre en médecine. Pédagogie Médicale 2001;2:235-41.

Correspondance et offprints : Guillaume Lebreton, « La Rivière », 50530 Dragey, France

Mailto : guillaumelebreton@live.fr 